\title{
ON ALMOST PERIODIC SOLUTIONS OF THE COMPETING SPECIES PROBLEMS
}

\author{
SHAIR AHMAD
}

(Communicated by George Sell)

\begin{abstract}
This paper considers the two-dimensional Volterra-Lotka competition equations which are almost periodic in time. Conditions for the existence of an asymptotically stable almost periodic solution with positive components are given.
\end{abstract}

Introduction. The main motivation for this work comes from a recent paper by K. Gopalsamy [5], who considers the system of $n(n \geq 2)$ differential equations

$$
x_{i}^{\prime}(t)=x_{i}(t)\left[b_{i}(t)-\sum_{j=1}^{m} a_{i j}(t) x_{j}(t)\right],
$$

$i=1, \ldots, n$, where it is assumed that the functions $b_{i}$ and $a_{i j}$ are positive, continuous, bounded below by positive constants, and almost periodic on $(-\infty, \infty)$. In [5] it was shown that if $a_{i j L}, a_{i j M}\left(b_{i L}, b_{i M}\right)$ denote the inf and sup of $a_{i j}(t)$, $i, j=1, \ldots, n\left(b_{i}(t), i=1, \ldots, n\right)$, respectively, then the two sets of conditions

$$
b_{i L}>\sum_{\substack{j=1 \\ j \neq i}}^{n} a_{i j M}\left(b_{j M} / a_{j j L}\right), \quad i=1, \ldots, n,
$$

and

$$
a_{i i L}>\sum_{\substack{j=1 \\ j \neq i}}^{n} a_{j i M}, \quad i=1, \ldots, n,
$$

imply that the system $(\mathrm{S})$ has a solution $\operatorname{col}\left(x_{10}, \ldots, x_{n 0}\right)$ such that each component is almost periodic and bounded below by a positive constant on $(-\infty, \infty)$. Moreover, if $\operatorname{col}\left(x_{1}(t), \ldots, x_{n}(t)\right)$ is a solution of $(\mathrm{S})$ such that $x_{i}\left(t_{0}\right)>0$ for some $t_{0}$, then $\lim _{t \rightarrow \infty}\left[x_{i}(t)-x_{i 0}(t)\right]=0$ for $1 \leq i \leq n$.

The ecological significance of such a system is discussed in [5].

It is easy to see, by considering the autonomous case for example, that conditions $\left(G_{1}\right)$ and $\left(G_{2}\right)$ are independent. In this paper we show that if $n=2$ then conditions $\left(\mathrm{G}_{1}\right)$ alone imply the assertion of the above-mentioned theorem of Gopalsamy. In [1] the author showed that if $n=2$ and the functions $a_{i j}(t), 1 \leq i, j \leq 2$, and $b_{i}(t)$ are merely assumed to be continuous and bounded above and below by positive

Received by the editors December 4, 1986.

1980 Mathematics Subject (lassification (1985 Revision). Primary 34C27; Secondary 34D05.

Key words and phrases. Almost periodic, positive components, bounded, Volterra-Lotka.

Acknowledgment. This research was supported by the University of West Florida. 
constants on an interval $\left[t_{0}, \infty\right)$, then conditions $\left(G_{1}\right)$ imply that the differences of the corresponding components of two solutions of $(S)$, both of whose components are positive at $t_{0}$, tend to zero as $t \rightarrow \infty$. To prove the above claim, it is therefore sufficient to show that if $n=2$ and the functions are almost periodic and bounded above and below by positive constants, then conditions $\left(\mathrm{G}_{1}\right)$ imply the existence of an almost periodic solution both of whose components are bounded below by positive constants. This is the content of Theorem 2 of this note.

The periodic case under conditions $\left(\mathrm{G}_{1}\right)$ with $n=2$ was treated in [2] but by completely different methods.

In regard to the theory of almost periodic functions, this paper is self-contained since the only fact from the theory which we use is Bochner's criterion for almost periodicity which we may take as a definition (see, for example, [4]). This says that a function $g(t)$, continuous on $-\infty<t<\infty$, is almost periodic if and only if for every sequence of numbers $\left\{\tau_{m}\right\}_{1}^{\infty}$, there exists a subsequence $\left\{\tau_{m_{k}}\right\}_{k=1}^{\infty}$ such that the sequence of translates $\left\{g\left(t+\tau_{m_{k}}\right)\right\}_{k=1}^{\infty}$ converges uniformly on $(-\infty, \infty)$.

We also use ideas from a classic paper by Amerio [3] on almost periodic systems of differential equations, although we do not use his theorem directly. We first consider the system ( $\mathrm{S}$ ) for $n=2$, where it is only assumed that the functions be continuous and bounded above and below by positive constants on $(-\infty, \infty)$.

Existence of an almost periodic solution. Consider the system of differential equations

$$
\left\{\begin{array}{l}
u^{\prime}=u[a(t)-b(t) u(t)-c(t) v(t)], \\
v^{\prime}=v[d(t)-e(t) u(t)-f(t) v(t)],
\end{array}\right.
$$

where the functions $a(t), b(t), c(t), d(t), e(t)$ and $f(t)$ are continuous and bounded above and below by positive constants. Given a function $g(t)$, we let $g_{L}$ and $g_{M}$ denote inf $-\infty<t<\infty g(t)$ and $\sup _{-\infty<t<\infty} g(t)$, respectively. Throughout this paper we assume the inequalities $a_{L}>c_{M} d_{M} / f_{L}$ and $d_{L}>e_{M} a_{M} / b_{L}$.

As in [1], let $\varepsilon, k_{1}, k_{2}$ be numbers satisfying the inequalities

$$
\begin{gathered}
0<\varepsilon<k_{1}, k_{2}, \quad a_{M / b_{L}}<k_{1}, \quad d_{M / f_{L}}<k_{2}, \\
d_{L}-e_{M} k_{1}-f_{M} \varepsilon>0, \quad a_{L}-b_{M} \varepsilon-c_{M} k_{2}>0 .
\end{gathered}
$$

Let $S=\left\{\operatorname{col}(\xi, \eta) \mid \varepsilon \leq \xi \leq k_{1}, \varepsilon \leq \eta \leq k_{2}\right\}$.

THEOREM 1. There exists a unique solution $\operatorname{col}\left(u_{0}(t), v_{0}(t)\right)$ of $(1)$ defined on $(-\infty, \infty)$, with $\operatorname{col}\left(u_{0}(t), v_{0}(t)\right) \in S$ for all $t$ in $(-\infty, \infty)$.

The proof of Theorem 1 follows from the following two lemmas.

LEMMA 1. There exist solutions $\operatorname{col}\left(u^{*}(t), v^{*}(t)\right)$ and $\operatorname{col}\left(u_{*}(t), v_{*}(t)\right)$, defined on $(-\infty, \infty)$, with $\varepsilon \leq u_{*}(t) \leq u^{*}(t) \leq k_{1}$ and $\varepsilon \leq v^{*}(t) \leq v_{*}(t) \leq k_{2}$, and such that if $\operatorname{col}(\hat{u}(t), \hat{v}(t))$ is any other solution with $\operatorname{col}(\hat{u}(t), \hat{v}(t)) \in S$ for all $t$, then $u_{*}(t) \leq \hat{u}(t) \leq u^{*}(t)$ and $v^{*}(t) \leq \hat{v}(t) \leq v_{*}(t)$.

PROOF. In [1] it was shown that if $\operatorname{col}\left(u_{1}(t), v_{1}(t)\right)$ and $\operatorname{col}\left(u_{2}(t), v_{2}(t)\right)$ are two solutions with $u_{1}\left(t_{0}\right)=k_{1}, v_{1}\left(t_{0}\right)=\varepsilon, u_{2}\left(t_{0}\right)=\varepsilon$, and $v_{2}\left(t_{0}\right)=k_{2}$ for some $t_{0}$ in $(-\infty, \infty)$, then $\varepsilon \leq u_{2}(t) \leq u_{1}(t) \leq k_{1}$ and $\varepsilon \leq v_{1}(t) \leq v_{2}(t) \leq k_{2}$ for all $t \geq t_{0}$. 
For each integer $n, n=1,2, \ldots$, let $\operatorname{col}\left(u_{1 n}(t), v_{1 n}(t)\right)$ and $\operatorname{col}\left(u_{2 n}(t), v_{2 n}(t)\right)$ be the solutions satisfying

$$
\operatorname{col}\left(u_{1 n}(-n), v_{1 n}(-n)\right)=\operatorname{col}\left(k_{1}, \varepsilon\right) \quad \text { and } \operatorname{col}\left(u_{2 n}(-n), v_{2 n}(-n)\right)=\operatorname{col}\left(\varepsilon, k_{2}\right) .
$$

By applying the previous result, it follows that $\operatorname{col}\left(u_{k n}(t), v_{k n}(t)\right)$ is defined for $-n \leq t<\infty, k=1,2, \varepsilon \leq u_{2 n}(t) \leq u_{1 n}(t) \leq k_{1}$, and $\varepsilon \leq v_{1 n}(t) \leq v_{2 n}(t) \leq k_{2}$ for all $t \geq-n$. Since we have $\varepsilon \leq u_{2 n}(0) \leq u_{1 n}(0) \leq k_{1}$ and $\varepsilon \leq v_{1 n}(0) \leq v_{2 n}(0) \leq k_{2}$ for all $n$, it follows that there exists a sequence of integers $\left\{n_{j}\right\}_{j=1}^{\infty}$ such that the sequences $\left\{u_{1 n_{j}}(0)\right\},\left\{u_{2 n_{j}}(0)\right\},\left\{v_{1 n_{j}}(0)\right\}$ and $\left\{v_{2 n_{j}}(0)\right\}$ converge to numbers $\xi^{*}, \xi_{*}, \eta_{*}$ and $\eta^{*}$, respectively, satisfying $\varepsilon \leq \xi_{*} \leq \xi^{*} \leq k_{1}$ and $\varepsilon \leq \eta^{*} \leq \eta_{*} \leq k_{2}$. Let $\operatorname{col}\left(u^{*}(t), v^{*}(t)\right)$ and $\operatorname{col}\left(u_{*}(t), v_{*}(t)\right)$ be the solutions satisfying

$$
\operatorname{col}\left(u^{*}(0), v^{*}(0)\right)=\operatorname{col}\left(\xi^{*}, \eta^{*}\right) \quad \text { and } \quad \operatorname{col}\left(u_{*}(0), v_{*}(0)\right)=\operatorname{col}\left(\xi_{*}, \eta_{*}\right) .
$$

Now, we wish to show that $\operatorname{col}\left(u^{*}(t), v^{*}(t)\right)$ and $\operatorname{col}\left(u_{*}(t), v_{*}(t)\right)$ satisfy the assertions of the lemma. If $\operatorname{dom}\left(\operatorname{col}\left(u^{*}(t), v^{*}(t)\right)\right) \neq(-\infty, \infty)$, then there must exist a number $t_{0}$ in the domain of $\operatorname{col}\left(u^{*}(t), v^{*}(t)\right)$ such that $\operatorname{col}\left(u^{*}\left(t_{0}\right), v^{*}\left(t_{0}\right)\right) \notin S$. Since $\operatorname{col}\left(u_{1 n_{j}}(0), v_{1 n_{j}}(0)\right) \rightarrow \operatorname{col}\left(u^{*}(0), v^{*}(0)\right)$ as $j \rightarrow \infty$, it follows that

$$
\left.\operatorname{col}\left(u_{1 n_{j}}(t), v_{1 n_{j}}(t)\right) \rightarrow \operatorname{col}\left(u^{*}(t), v^{*}(t)\right)\right)
$$

uniformly on compact subsets of the domain of $\operatorname{col}\left(u^{*}(t), v^{*}(t)\right)$. In particular,

$$
\operatorname{col}\left(u_{1 n_{j}}\left(t_{0}\right), v_{1 n_{j}}\left(t_{0}\right)\right) \rightarrow \operatorname{col}\left(u^{*}\left(t_{0}\right), v^{*}\left(t_{0}\right)\right) \quad \text { as } j \rightarrow \infty .
$$

Hence, $\operatorname{col}\left(u_{1 n_{j}}\left(t_{0}\right), v_{1 n_{j}}\left(t_{0}\right)\right) \notin S$ for $j$ large enough. But, by construction, $\varepsilon \leq$ $u_{1 n_{j}}(t) \leq k_{1}$ and $\varepsilon \leq v_{1 n_{j}}(t) \leq k_{2}$ on the interval $\left(-n_{j}, \infty\right)$, which implies that $\operatorname{col}\left(u_{1 n_{j}}\left(t_{0}\right), v_{1 n_{j}}\left(t_{0}\right)\right) \in S$ if $-n_{j}<t_{0}$; a contradiction. This shows that

$$
\operatorname{dom}\left(\operatorname{col}\left(u^{*}(t), v^{*}(t)\right)\right)=(-\infty, \infty) .
$$

Similarly,

$$
\operatorname{dom}\left(\operatorname{col}\left(u_{*}(t), v_{*}(t)\right)\right)=(-\infty, \infty) .
$$

We note that for a given number $\hat{t}$, by continuity with respect to initial conditions, we have $\lim _{j \rightarrow \infty} u_{1 n_{j}}(\hat{t})=u^{*}(\hat{t}), \lim _{j \rightarrow \infty} v_{1 n_{j}}(\hat{t})=v^{*}(\hat{t}), \lim _{j \rightarrow \infty} u_{2 n_{j}}(\hat{t})=$ $u_{*}(\hat{t})$, and $\lim _{j \rightarrow \infty} v_{2 n_{j}}(\hat{t})=v_{*}(\hat{t})$. Also, $\varepsilon \leq u_{2 n_{j}}(\hat{t}) \leq u_{1 n_{j}}(\hat{t}) \leq k_{1}$, and $\varepsilon \leq$ $v_{1 n_{j}}(\hat{t}) \leq v_{2 n_{j}}(\hat{t}) \leq k_{2}$ if $-n_{j} \leq \hat{t}$. Letting $j \rightarrow \infty$, we obtain $\varepsilon \leq u_{*}(\hat{t}) \leq u^{*}(\hat{t}) \leq k_{1}$ and $\varepsilon \leq v^{*}(\hat{t}) \leq v_{*}(\hat{t}) \leq k_{2}$.

Next, assume that $\operatorname{col}(\hat{u}(t), \hat{v}(t))$ is a solution of (1) satisfying the inequalities $\varepsilon \leq \hat{u}(t) \leq k_{1}$ and $\varepsilon \leq \hat{v}(t) \leq k_{2}$. Let $t_{0}$ be an arbitrary number. Then, for $-n_{j}<t_{0}$, we have $u_{2 n_{j}}\left(-n_{j}\right)=\varepsilon \leq \hat{u}\left(-n_{j}\right)$ and $\hat{v}\left(-n_{j}\right) \leq k_{2}=v_{2 n_{j}}\left(-n_{j}\right)$. It follows from our earlier comparison result (see [1]) that $u_{2 n_{j}}(t) \leq \hat{u}(t)$ and $\hat{v}(t) \leq v_{2 n_{j}}(t)$ if $-n_{j} \leq t<\infty$. In particular, $u_{2 n_{j}}\left(t_{0}\right) \leq \hat{u}\left(t_{0}\right)$ and $\hat{v}\left(t_{0}\right) \leq v_{2 n_{j}}\left(t_{0}\right)$. By the first part of the proof, we have

$$
\lim _{j \rightarrow \infty} \operatorname{col}\left(u_{2 n_{j}}\left(t_{0}\right), v_{2 n_{j}}\left(t_{0}\right)\right)=\operatorname{col}\left(u_{*}\left(t_{0}\right), v_{*}\left(t_{0}\right)\right) .
$$

Consequently, $u_{*}\left(t_{0}\right) \leq \hat{u}\left(t_{0}\right)$ and $\hat{v}\left(t_{0}\right) \leq v_{*}\left(t_{0}\right)$. The inequalities $\hat{u}(t) \leq u^{*}(t)$ and $v^{*}(t) \leq \hat{v}(t)$ follow similarly. 
LEMMA 2. Let $\operatorname{col}\left(u^{*}(t), v^{*}(t)\right)$ and $\operatorname{col}\left(u_{*}(t), v_{*}(t)\right)$ be as in Lemma 1. Then, $\operatorname{col}\left(u^{*}(t), v^{*}(t)\right)=\operatorname{col}\left(u_{*}(t), v_{*}(t)\right)$ for all $t$ in $(-\infty, \infty)$.

ProOF. We note that

$$
\frac{u^{* \prime}(t)}{u^{*}(t)}-\frac{u_{*}^{\prime}(t)}{u_{*}(t)}=-b(t)\left(u^{*}(t)-u_{*}(t)\right)-c(t)\left(v^{*}(t)-v_{*}(t)\right)
$$

Similarly,

$$
\frac{v_{*}^{\prime}(t)}{v_{*}(t)}-\frac{v^{* \prime}(t)}{v^{*}(t)}=-e(t)\left(u_{*}(t)-u^{*}(t)\right)-f(t)\left(v_{*}(t)-v^{*}(t)\right)
$$

Consequently, we have

$$
\begin{aligned}
& \frac{d}{d t} \ln \left(\frac{u^{*}(t)}{u_{*}(t)}\right)=-b(t)\left(u^{*}(t)-u_{*}(t)\right)-c(t)\left(v^{*}(t)-v_{*}(t)\right), \\
& \frac{d}{d t} \ln \left(\frac{v_{*}(t)}{v^{*}(t)}\right)=-e(t)\left(u_{*}(t)-u^{*}(t)\right)-f(t)\left(v_{*}(t)-v^{*}(t)\right) .
\end{aligned}
$$

Therefore,

$$
\begin{gathered}
\frac{d}{d t} \ln \left(\frac{u^{*}(t)}{u_{*}(t)}\right) \leq-b_{L}\left(u^{*}(t)-u_{*}(t)\right)+c_{M}\left(v_{*}(t)-v^{*}(t)\right), \\
\frac{d}{d t} \ln \left(\frac{v_{*}(t)}{v^{*}(t)}\right) \leq e_{M}\left(u^{*}(t)-u_{*}(t)\right)=f_{L}\left(v_{*}(t)-v^{*}(t)\right)
\end{gathered}
$$

Multiplying (2) by $f_{L}$ and (3) by $c_{M}$, and adding, we obtain

(4) $\frac{d}{d t}\left[f_{L} \ln \left(\frac{u^{*}(t)}{u_{*}(t)}\right)+c_{M} \ln \left(\frac{v_{*}(t)}{v^{*}(t)}\right)\right] \leq\left(c_{M} e_{M}-f_{L} b_{L}\right)\left(u^{*}(t)-u_{*}(t)\right)$.

Let $\Delta=f_{L} b_{L}-c_{M} e_{M}$. It follows that $\Delta>0$, since

$$
a_{L}>\frac{c_{M} d_{M}}{f_{L}} \geq\left(\frac{c_{M}}{f_{L}}\right) d_{L}>\frac{c_{M}}{f_{L}} \cdot \frac{e_{M}}{b_{L}} \cdot a_{M} \geq \frac{c_{M} e_{M}}{f_{L} b_{L}} \cdot a_{L}
$$

Therefore, (4) can be written as

$$
u^{*}(t)-u_{*}(t) \leq-\frac{1}{\Delta} \frac{d}{d t}\left[f_{L} \ln \left(\frac{u^{*}(t)}{u_{*}(t)}\right)+c_{M} \ln \left(\frac{v_{*}(t)}{v^{*}(t)}\right)\right] .
$$

Similarly, multiplying (2) by $e_{M}$ and (3) by $b_{L}$, and adding, we obtain

$$
v_{*}(t)-v^{*}(t) \leq \frac{1}{\Delta} \frac{d}{d t}\left[e_{M} \ln \left(\frac{u^{*}(t)}{u_{*}(t)}\right)+b_{L} \ln \left(\frac{v_{*}(t)}{v^{*}(t)}\right)\right] .
$$

Since the ln function is increasing, we have

$$
0 \leq \ln \left(\frac{u^{*}(t)}{u_{*}(t)}\right) \leq \ln \left(\frac{k_{1}}{\varepsilon}\right), \quad 0 \leq \ln \left(\frac{v_{*}(t)}{v^{*}(t)}\right) \leq \ln \left(\frac{k_{2}}{\varepsilon}\right) .
$$

Therefore, it follows from (5) that for any fixed number $T>0$,

$$
0 \leq \int_{-T}^{T}\left(u^{*}(t)-u_{*}(t)\right) d t \leq M_{1}
$$


where $M_{1}$ is some number independent of $t$. Hence $\int_{-\infty}^{\infty}\left(u^{*}(t)-u_{*}(t)\right) d t \leq M_{1}<$ $\infty$. Similarly, $\int_{-\infty}^{\infty}\left(v_{*}(t)-v^{*}(t)\right) d t \leq M_{2}<\infty$ for some number $M_{2}$.

We wish to show that $u^{*}(t)-u_{*}(t) \rightarrow 0$ and $v_{*}(t)-v^{*}(t) \rightarrow 0$ as $t \rightarrow \pm \infty$. If $w(t)=u^{*}(t)-u_{*}(t)$, then it follows from the form of the differential equation (1) and the boundedness of the functions $a(t), \ldots, f(t)$ that $w^{\prime}(t)$ is bounded. It follows that $\int_{-\infty}^{0} w^{\prime}(t) w(t) d t$ and, hence, $\lim _{T \rightarrow \infty} \int_{-T}^{0} 2 w^{\prime}(t) w(t) d t$ exists. We note that $\int_{-T}^{0} 2 w^{\prime}(t) w(t) d t=w(0)^{2}-w(-T)^{2}$ implies that $\lim _{T \rightarrow \infty} w(-T)$ exists. Therefore, since $\int_{-\infty}^{0} w(t) d t$ exists, we must have $\lim _{T \rightarrow \infty} w(-T)=0$.

A similar argument can be applied to $\int_{0}^{\infty} w^{\prime}(t) w(t) d t$. Since

$$
\frac{u^{*}(t)-u_{*}(t)}{k_{1}} \leq \frac{u^{*}(t)-u_{*}(t)}{u^{*}(t)} \leq \frac{u^{*}(t)-u_{*}(t)}{\varepsilon},
$$

it follows that $\lim _{s \rightarrow \pm \infty}\left(\left(u^{*}(s)-u_{*}(s)\right) / u_{*}(s)\right)=0$. Consequently,

$$
\lim _{s \rightarrow \pm \infty}\left(u^{*}(s) / u_{*}(s)\right)=1, \quad \text { and } \quad \lim _{s \rightarrow \pm \infty} \ln \left(u^{*}(s) / u_{*}(s)\right)=0 .
$$

Similarly, $\lim _{s \rightarrow \pm \infty} \ln \left(v_{*}(s) / v^{*}(s)\right)=0$. It follows from (5) and (6) that

$$
\int_{-\infty}^{\infty}\left(u^{*}(t)-u_{*}(t)\right) d t=0=\int_{-\infty}^{\infty}\left(v_{*}(t)-v^{*}(t)\right) d t .
$$

Hence, $u^{*}(t) \equiv u_{*}(t)$ and $v_{*}(t) \equiv v^{*}(t)$.

THEOREM 2. Assume that the functions $a(t), b(t), c(t), d(t), e(t)$ and $f(t)$ are almost periodic. If $a_{L}>c_{M} d_{M} / f_{L}$ and $d_{L}>e_{M} a_{M} / b_{L}$, then the unique solution $\operatorname{col}\left(u_{0}(t), v_{0}(t)\right)$ of Theorem 1 is almost periodic.

PROOF. Let $\left\{\tau_{m}\right\}_{m=1}^{\infty}$ be an arbitrary sequence of numbers. We wish to show that there exists a subsequence $\left\{\tau_{m_{K}}\right\}$ of $\left\{\tau_{m}\right\}$ such that the sequence $\left\{u_{0}\left(t+\tau_{m_{k}}\right), v_{0}\left(t+\tau_{m_{k}}\right)\right\}$ converges uniformly on $(-\infty, \infty)$. We let \|\| denote the Euclidean norm.

Since $a(t), b(t), \ldots, f(t)$ are almost periodic, there exists a subsequence $\left\{\tau_{m_{k}}\right\}$ of $\left\{\tau_{m}\right\}$ such that $\left\{a\left(t+\tau_{m_{k}}\right)\right\}, \ldots,\left\{f\left(t+\tau_{m_{k}}\right)\right\}$ converge uniformly to functions $a^{*}(t), \ldots, f^{*}(t)$, respectively, on $(-\infty, \infty)$. It is easy to see that $a_{L}^{*}=a_{L}, a_{M}^{*}=$ $a_{M}, \ldots, f_{L}^{*}=f_{L}$, and $f_{M}^{*}=f_{M}$.

It follows from Theorem 1 that the system

$$
\left\{\begin{array}{l}
u^{\prime}(t)=u(t)\left[a^{*}(t)-b^{*}(t) u(t)-c^{*}(t) v(t)\right], \\
v^{\prime}(t)=v(t)\left[d^{*}(t)-e^{*}(t) u(t)-f^{*}(t) v(t)\right]
\end{array}\right.
$$

has a solution $\operatorname{col}\left(u_{0}^{*}(t), v_{0}^{*}(t)\right)$ defined on $(-\infty, \infty)$ such that $\left(u_{0}^{*}(t), v_{0}^{*}(t)\right) \in S$ for all $t$ in $(-\infty, \infty)$. We claim that $\left(u_{0}\left(t+\tau_{m_{k}}\right), v_{0}\left(t+\tau_{m_{k}}\right)\right) \rightarrow\left(u_{0}^{*}(t), v_{0}^{*}(t)\right)$ uniformly as $k \rightarrow \infty$, which will show that $\operatorname{col}\left(u_{0}(t), v_{0}(t)\right)$ is almost periodic. Suppose that the claim is false. Then there exists a subsequence $\left\{\tau_{m_{k_{j}}}\right\}$ of $\left\{\tau_{m_{k}}\right\}$, a sequence of numbers $\left\{s_{j}\right\}$, and a fixed number $\alpha>0$ such that

$$
\left\|\left(u_{0}\left(s_{j}+\tau_{m_{k_{j}}}\right), v_{0}\left(s_{j}+\tau_{m_{k_{j}}}\right)\right)-\left(u_{0}^{*}\left(s_{j}\right), v_{0}^{*}\left(s_{j}\right)\right)\right\| \geq \alpha, \quad \text { for all } j .
$$

Since the functions $a(t), \ldots, f(t)$ are almost periodic, we may assume, without loss of generality, that $a\left(t+\tau_{m_{k_{j}}}+s_{j}\right) \rightarrow \hat{a}(t), \ldots, f\left(t+\tau_{m_{k_{j}}}+s_{j}\right) \rightarrow \hat{f}(t)$ as $j \rightarrow \infty$, 
uniformly with respect to $t$ in $(-\infty, \infty)$. It follows that $a^{*}\left(t+s_{j}\right) \rightarrow \hat{a}(t), \ldots$, $f^{*}\left(t+s_{j}\right) \rightarrow \hat{f}(t)$ as $j \rightarrow \infty$, uniformly with respect to $t$ in $(-\infty, \infty)$, and hence $\hat{a}_{L}=a_{L}, \hat{a}_{M}=a_{M}, \ldots, \hat{f}_{L}=f_{L}$, and $\hat{f}_{M}=f_{M}$. Since $\operatorname{col}\left(u_{0}(t), v_{0}(t)\right) \in S$ for all $t$ in $(-\infty, \infty)$, we can assume without loss of generality that $\left(u_{0}\left(s_{j}+\tau_{m_{k_{j}}}\right)\right.$, $\left.v_{0}\left(s_{j}+\tau_{m_{k_{j}}}\right)\right) \rightarrow\left(\xi_{0}, \eta_{0}\right)$ as $j \rightarrow \infty$, where $\left(\xi_{0}, \eta_{0}\right) \in S$. Similarly, we may assume that $\left(u_{0}^{*}\left(s_{j}\right), v_{0}^{*}\left(s_{j}\right)\right) \rightarrow\left(\xi_{0}^{*}, \eta_{0}^{*}\right)$ as $j \rightarrow \infty$. Clearly, $\left\|\left(\xi_{0}, \eta_{0}\right)-\left(\xi_{0}^{*}, \eta_{0}^{*}\right)\right\| \geq \alpha$.

For each integer $j, j=1,2, \ldots, \operatorname{col}\left(u_{0}\left(t+\tau_{m_{k_{j}}}+s_{j}\right), v_{0}\left(t+\tau_{m_{k_{j}}}+s_{j}\right)\right)$ is a solution of the system

$$
\left\{\begin{array}{l}
u^{\prime}=u\left[a\left(t+\tau_{m_{k_{j}}}+s_{j}\right)-b\left(t+\tau_{m_{k_{j}}}+s_{j}\right) u-c\left(t+\tau_{m_{k_{j}}}+s_{j}\right) v\right] \\
v^{\prime}=v\left[d\left(t+\tau_{m_{k_{j}}}+s_{j}\right)-e\left(t+\tau_{m_{k_{j}}}+s_{j}\right) u-f\left(t+\tau_{m_{k_{j}}}+s_{j}\right) v\right] .
\end{array}\right.
$$

Consider the solution $\operatorname{col}\left(\hat{u}_{0}, \hat{v}_{0}\right)$ of

$$
\left\{\begin{array}{l}
u^{\prime}=u[\hat{a}(t)-\hat{b}(t) u-\hat{c}(t) v] \\
v^{\prime}=v[\hat{d}(t)-\hat{e}(t) u-\hat{f}(t) v]
\end{array}\right.
$$

having the initial value $\operatorname{col}\left(\hat{u}_{0}(0), \hat{v}_{0}(0)\right)=\operatorname{col}\left(\xi_{0}, \eta_{0}\right)$. We have two systems

$$
\left\{\begin{array}{l}
u^{\prime}=f(t, u, v) \\
v^{\prime}=g(t, u, v)
\end{array}\right.
$$

and

$$
\left\{\begin{array}{l}
u^{\prime}=f_{j}(t, u, v) \\
v^{\prime}=g_{j}(t, u, v)
\end{array}\right.
$$

where the right side of $\left(1_{j}\right)$ converges uniformly to the right side of $\left(1^{\prime}\right)$ on compact subsets of $R^{3}$, as $j \rightarrow \infty$. Also the initial values satisfy the property that $\operatorname{col}\left(u_{0}\left(\tau_{m_{k_{j}}}+s_{j}\right), v_{0}\left(\tau_{m_{k_{j}}}+s_{j}\right)\right) \rightarrow \operatorname{col}\left(\xi_{0}, \eta_{0}\right)$. Hence it follows that

$$
\left(u_{0}\left(t+\tau_{m_{k_{j}}}+s_{j}\right), v_{0}\left(t+\tau_{m_{k_{j}}}+s_{j}\right)\right) \rightarrow\left(\hat{u}_{0}(t), \hat{v}_{0}(t)\right)
$$

uniformly on compact subintervals of the domain of $\operatorname{col}\left(\hat{u}_{0}(t), \hat{v}_{0}(t)\right)$. This implies that $\left(\hat{u}_{0}(t), \hat{v}_{0}(t)\right) \in S$ for all $t$.

Now, recall that $\operatorname{col}\left(u_{0}^{*}, v_{0}^{*}\right)$ is the unique solution of $(7)$ with $\left(u_{0}^{*}(t), v_{0}^{*}(t)\right) \in S$ for all $t$. For each integer $j, \operatorname{col}\left(u_{0}^{*}\left(t+s_{j}\right), v_{0}^{*}\left(t+s_{j}\right)\right)$ is a solution of

$$
\left\{\begin{array}{l}
u^{\prime}=u\left[a^{*}\left(t+s_{j}\right)-b^{*}\left(t+s_{j}\right) u-c^{*}\left(t+s_{j}\right) v\right], \\
v^{\prime}=v\left[d^{*}\left(t+s_{j}\right)-e^{*}\left(t+s_{j}\right) u-f^{*}\left(t+s_{j}\right) v\right],
\end{array}\right.
$$

with $\left(u_{0}^{*}\left(s_{j}\right), v_{0}^{*}\left(s_{j}\right)\right) \rightarrow\left(\xi_{0}^{*}, \eta_{0}^{*}\right)$ as $j \rightarrow \infty$. Since $a^{*}\left(t+s_{j}\right) \rightarrow \hat{a}(t), \ldots, f^{*}\left(t+s_{j}\right) \rightarrow$ $\hat{f}(t)$ as $j \rightarrow \infty$, uniformly with respect to $t$, it follows that if $\operatorname{col}\left(\hat{u}_{0}^{*}, \hat{v}_{0}^{*}\right)$ is the solution of $\left(1^{\prime}\right)$ with $\operatorname{col}\left(\hat{u}_{0}^{*}(0), \hat{v}_{0}^{*}(0)\right)=\operatorname{col}\left(\xi_{0}^{*}, \eta_{0}^{*}\right)$, then $\left(u_{0}^{*}\left(t+s_{j}\right), v_{0}^{*}\left(t+s_{j}\right)\right) \rightarrow$ $\left(\hat{u}^{*}(t), \hat{v}_{0}^{*}(t)\right)$ uniformly on compact subintervals of the domain of $\operatorname{col}\left(\hat{u}_{0}^{*}, \hat{v}_{0}^{*}\right)$. By the same argument given before, we have $\left(\hat{u}_{0}^{*}(t), \hat{v}_{0}^{*}(t)\right) \in S$ for all $t$. We also have $\left(\hat{u}_{0}(t), \hat{v}_{0}(t)\right) \in S$ for all $t$. Since both are solutions of $\left(1^{\prime}\right)$, and since $\hat{a}_{L}=a_{L}, \hat{a}_{M}=$ $a_{M}, \ldots, \hat{f}_{L}=f_{L}$, and $\hat{f}_{M}=f_{M}$, we must have $\left(\hat{u}_{0}(t), \hat{v}_{0}(t)\right) \equiv\left(\hat{u}^{*}(t), \hat{v}_{0}^{*}(t)\right)$ by Theorem 1. But, $\left(\hat{u}_{0}(0), \hat{v}_{0}(0)\right)=\left(\xi_{0}, \eta_{0}\right),\left(\hat{u}_{0}^{*}(0), \hat{v}_{0}^{*}(0)\right)=\left(\xi_{0}^{*}, \eta_{0}^{*}\right)$, and $\|\left(\xi_{0}, \eta_{0}\right)-$ $\left(\xi_{0}^{*}, \eta_{0}^{*}\right) \| \geq \alpha$, which is a contradiction. This proves the theorem.

ACKNOWLEDGMENT. The author wishes to thank the referee for pointing out that the proof of Theorem 2 follows from Theorem 1 of the author's and Theorems 1 and 2 of Sacker-Sell [6]. However, for the sake of completeness and easy accessibility, we include the elementary proof of Theorem 2. 


\section{REFERENCES}

1. S. Ahmad, Convergence and ultimate bounds of solutions of the nonautonomous Volterra-Lotka competition equations, J. Math. Anal. Appl. 127 (1987).

2. C. Alvarez and A. C. Lazer, An application of topological degree to the periodic competing species problem, J. Austral. Math. Soc. Ser. B 28 (1986).

3. L. Amerio, Soluzioni quasiperiodiche, o limite di sistemi differenziali quasiperiodici o limitati, Ann. Mat. Pura Appl. 34 (1955), 97-116.

4. A. S. Besicovitch, Almost periodic functions, Cambridge Univ. Press, 1932.

5. K. Gopalsamy, Global asymptotic stability in an almost periodic Lotka-Volterra system, J. Austral. Math. Soc. Ser. B 27 (1986), 346-360.

6. R. J. Sacker and G. R. Sell, Lifting properties in skew-product flows with applications to differential equations, Mem. Amer. Math. Soc. No. 190 (1977).

DEPARTMENT OF MATHEMATICS AND COMPUTER SCIENCE, UNIVERSity OF Miami, CORAL GABLES, FLORIDA 33124 